Research Article

\title{
Thromboelastogram-Guided Transfusion Therapy Reduces Blood-Component Transfusion and Improves Coagulation Function during Orthopedic Surgery
}

\author{
Yan Zhang, Yue Song, Yixin Zhang, Lu Yu, and Kai Zhang $\mathbb{D}$ \\ Department of Blood Transfusion, Tianjin Hospital, No. 406, Jiefang South Road, Hexi District, Tianjin 300211, China \\ Correspondence should be addressed to Kai Zhang; xstltwz2021@163.com
}

Received 18 May 2021; Revised 8 June 2021; Accepted 10 June 2021; Published 28 June 2021

Academic Editor: Songwen Tan

Copyright (c) 2021 Yan Zhang et al. This is an open access article distributed under the Creative Commons Attribution License, which permits unrestricted use, distribution, and reproduction in any medium, provided the original work is properly cited.

\begin{abstract}
Massive bleeding is a serious medical complication arising from trauma, surgery, and invasive procedures. In this case, timely and effective hemostasis must be applied to patients. The aim of this study is to compare transfusion therapies guided by thromboelastogram (TEG) and conventional coagulation tests (CCTs) during orthopedic surgery, focusing on blood-component transfusion and coagulation function of patients. The patients who underwent orthopedic surgery in our hospital from May 2019 to November 2020 were retrospectively analyzed. According to different transfusion-guiding strategies, the patients were assigned into the CCT group containing 214 patients and the TEG group containing 266 patients. The TEG group used fewer volumes of blood products including red blood cell (RBC) suspension, fresh-frozen plasma, cryoprecipitate, and apheresis platelets than the CCT group $(P<0.05)$. After orthopedic surgery, the hemoglobin $(\mathrm{Hb})$ and $\mathrm{RBC}$ counts were decreased, but the white blood cell (WBC) counts were increased in all patients receiving whether transfusion therapy guided by TEG or CCTs. Importantly, the TEG group exhibited fewer WBC counts concomitant with higher $\mathrm{Hb}$ and more RBC counts than the CCT group $(P<0.05)$. There was no significant difference on the platelet (PLT) counts between the two groups before and after orthopedic surgery $(P>0.05)$. Significant declines on thrombin time $(\mathrm{TT})$, partial activated thromboplastin time (PATT), prothrombin time $(\mathrm{PT})$, and d-dimer (D-D), along with an increase on fibrinogen (FIB) were observed in two groups after surgery. The TEG group showed reduced TT, PATT, PT, D-D, and elevated FIB compared to the CCT group $(P<0.05)$. Posttransfusion, the $K$ value (time to reach a certain clot strength) and $R$ value (coagulation reaction time) were decreased, the angle $(\alpha)$ value (clot formation rate), MA value (maximum amplitude), and CI (coagulation index) were increased in the TEG group $(P<0.05)$. When the liver function was assessed, it was found that the levels of alanine aminotransferase (ALT), aspartate aminotransferase (AST), and total serum bilirubin (TBIL) were increased significantly, and albumin (ALB) was decreased between the two groups after surgery, but the TEG group with lower levels of ALT, AST, and TBIL and a higher level of ALB than the CCT group $(P<0.05)$. With regard to the renal function, two groups had increased levels of serum creatinine (Scr) and blood urea nitrogen (BUN) with a declined uric acid (UA) level after surgery; however, the patients in the TEG group had lower levels of Scr and BUN and a higher level of UA compared to the CCT group $(P<0.05)$. In view of above data, TEG-guided transfusion therapy could reduce use of blood products, optimize blood components, and improve coagulation function for patients undergoing orthopedic surgery. TEG-guided transfusion therapy may prevent liver and renal dysfunction after orthopedic surgery.
\end{abstract}

\section{Introduction}

Massive blood lose is a risk for mortality and morbidity during orthopedic surgeries [1], including total hip replacement, total knee replacement [2], and spine surgery [3]. Massive bleeding is a serious and life-threatening manifestation, which can cause a variety of complications, including coagulation abnormalities, immunosuppression, infection, hypothermia, and organ damage related with massive transfusion of blood products $[4,5]$. Blood transfusion involves red blood cell (RBC) suspension, fresh-frozen plasma, cryoprecipitate, and apheresis platelets, which is critical to the patients with massive 
bleeding [6]. Conventional coagulation tests (CCTs), such as thrombin time (TT), partial activated thromboplastin time (PATT), prothrombin time (PT), and fibrinogen (FIB), are related to the dose and timing of transfusion $[7,8]$. Shortage of blood products makes accurate judgment for time of blood transfusion and requirements of blood components urgent. However, the present massive blood transfusion protocol is fixed infusion of RBC, fresh frozen plasma (FFP), and platelets (PLTs) at a ratio of $1: 1: 1$, likely leading to a huge waste. Thromboelastogram (TEG) has been advanced by Dr. Hartert in 1948 [9], which comprehensively analyzes the coagulation markers and represents the coagulation activity from coagulation cascade to fibrinolysis, which reflects the whole hemostasis process [10]. Studies have shown that TEG has been played a crucial role in the guidance of transfusion in cardiac surgeries for decades $[11,12]$. Present researchers have attempted to establish a transfusion strategy guided by TEG during the perioperative period. However, due to variations in sample size and individual differences, different conclusions have been reported. There is limited evidence supporting its application in orthopedic surgery, and thus this study attempts to compare transfusion therapies guided by TEG and CCTs during orthopedic surgery, with a specific focus on bloodcomponent transfusion and coagulation function of patients.

\section{Methods}

2.1. Patient Enrollment. From May 2019 to November 2020, patients who had undergone orthopedic surgery in our hospital were retrospectively studied and then divided into a TEG group $(n=266)$ and a CCT group $(n=214)$ in accordance with transfusion therapies guided by TEG or CCTs. All they belonged to class I-II according to the American Society of Aneshesiologists (ASA) Physical Status Classification System. Those patients were excluded due to use of anticoagulants in half a year, cognitive impairment, hematological diseases, coagulation dysfunction, hepatic and renal insufficiency, RH blood group, chronic alcoholics, pregnant women, and absence of complete clinical data. Patients in the TEG group consisted of 201 males and 66 females, aged from 13-85 years and with an average of age $(48.56 \pm 21)$ years and underwent orthopedic surgery for lumbar spinal stenosis $(n=55)$, cervical spondylosis $(n=50$ ) , total knee replacement $(n=55)$, fracture $(n=100)$, and lumbar disc herniation $(n=6)$. Patients in the CCT group comprised of 162 males and 52 females, aged from 13-84 years and with an average of age $(48.54 \pm 29)$ years and underwent orthopedic surgery for lumbar spinal stenosis $(n=44)$, cervical spondylosis $(n=40)$, total knee replacement $(n=43)$, fracture $(n=80)$, and lumbar disc herniation $(n=7)$. The baseline information between the two groups was not significant $(P>0.05)$.

2.2. Transfusion Therapy Guided by TEG or CCTs. The automatic coagulation analyzer SYSMEX CA 7000 (TOA Medical Electronics Co., Kobe, Japan) and the hematology analyzer (XN-3000, TOA Medical Electronics Co.) were used to examine PT (normal range [s]: 11.0-13.0), APTT (normal range [s]: 23.00-37.00), TT (normal range [s]: 12.0-19.0), and FIB (normal range [g/L]: 2.0-4.0) before and after transfusion. When the hemoglobin $(\mathrm{Hb})$ was lower than $70 \mathrm{~g} / \mathrm{L}$ and hematocrit (HTC) was less than $25 \%$, RBC suspension $(2$ $\mathrm{U})$ was transfused. If the PLT (normal range $\left[\times 10^{9} / \mathrm{L}\right]: 100$ 300) was lower than $50 \times 10^{9} / \mathrm{L}$, apheresis platelet $(10 \mathrm{U})$ was infused.

The TEG analyzer (TEG5000, Haemoscope, USA) was used to analyze $R$ time (coagulation reaction time), $K$ time (time to reach a certain clot strength), $\alpha$ value (clot formation rate), MA value (maximum amplitude), and CI (coagulation index). The normal $R$ time ranges from $5 \mathrm{~min}$ to $8 \mathrm{~min}$, reflecting the function of coagulation factors. Prolonged $R$ time indicates deficiency of coagulation factors, and shortened $R$ time indicates hypercoagulability. The normal $K$ time ranges from $1 \mathrm{~min}$ to $3 \mathrm{~min}$, depending on the levels of FIB, and anticoagulants could delay the $K$ time. The $\alpha$ value ranges from $53^{\circ}$ to $72^{\circ}$, reflecting the rate of clot formation during which FIB and PLTs are both involved. Once severe hypocoagulability occurs, clot amplitude fails to reach 20 $\mathrm{mm}$, and the $K$ time is undetectable. In this situation, the $\alpha$ value is more significant. A greater angle reflects faster clot formation. Prolonged $K$ time with low angle indicates risk of hypocoagulability and blooding. Shortened $K$ time with high angle indicates risk of hypercoagulability and clot. The normal MA value ranges from $54 \mathrm{~mm}$ to $72 \mathrm{~mm}$, which is affected by FIB (20\%) and PLT (80\%), suggesting the accumulation of PLT. A smaller MA value suggests blooding, dilute blood, reduced numbers of PLTs, depletion, or deficiency of coagulation factors. A greater MA value suggests arterial and venous thrombosis and hypercoagulability. The normal CI ranges from -3 to 3 and equals $-0.6516 r-$ $0.3772 \mathrm{~K}+0.1224 \mathrm{MA}+(-7.7922) . \mathrm{CI}>3$ suggests hypercoagulability, and $\mathrm{CI}<-3$ suggests hypocoagulability. When the $R$ value was more than $8 \mathrm{~min}$, fresh-frozen plasma (15 $\mathrm{mL} / \mathrm{kg}$ ) was infused. When the MA value was less than 50 $\mathrm{mm}$, the apheresis platelet $(10 \mathrm{U})$ was infused. The cryoprecipitate $(13 \mathrm{U})$ was infused if the angle value was more than $72^{\circ}$. The remaining blood products were transfused according to patients' condition and clinical experience. The usage of RBC suspension, fresh-frozen plasma, cryoprecipitate, and apheresis platelet was recorded.

\subsection{Measurements of Liver and Renal Function. Fasting} venous blood was collected into EDTA-contained tubes which were then centrifuged to extract the plasma. Blood sample collection was performed pretransfusion and posttransfusion. According to the instructions provided by commercial available kits, aminotransferase (ALT), aspartate aminotransferase (AST), total serum bilirubin (TBIL), albumin (ALB), serum creatinine $(\mathrm{Scr})$, blood urea nitrogen (BUN), and uric acid (UA) were examined by the enzyme linked immunosorbent assay pretransfusion and posttransfusion.

2.4. Data Analysis. SPSS 20.0 software was used to process the data. The counting data was represented by percentage and analyzed by the chi-square test. The measurement data was represented by mean \pm standard deviation and analyzed by 
TABLE 1: The usage of blood products between the TEG and CCT groups.

\begin{tabular}{lccccc}
\hline Group & \multirow{2}{*}{ RBC suspension (U) } & Fresh frozen plasma $(\mathrm{mL})$ & $\begin{array}{c}\text { Cryoprecipitate } \\
(\mathrm{U})\end{array}$ & $\begin{array}{c}\text { Apheresis platelet }(10 \\
\mathrm{U})\end{array}$ & \multirow{2}{*}{ Length of hospital stay $(\mathrm{d})$} \\
\hline TEG $(n=266)$ & $4.51 \pm 0.09^{\mathrm{a}}$ & $254.36 \pm 20.17^{\mathrm{a}}$ & $16.34 \pm 1.25^{\mathrm{a}}$ & $2.04 \pm 0.06^{\mathrm{a}}$ & $10.35 \pm 2.11^{\mathrm{a}}$ \\
CCT $(n=214$ & $6.09 \pm 0.04$ & $423.54 \pm 40.28$ & $28.64 \pm 2.31$ & $3.42 \pm 0.08$ & $11.17 \pm 2.23$ \\
) & & & & & \\
\hline
\end{tabular}

The letter of a suggests $P<0.05$ compared to the CCT group.

TABLE 2: Blood routine tests following TEG- or CCT-guided transfusion therapy.

\begin{tabular}{lccccc}
\hline Group & Time & Hb $(\mathrm{g} / \mathrm{L})$ & WBC $(\times 109 / \mathrm{L})$ & PLT $\left(\times 10^{9} / \mathrm{L}\right)$ & RBC $(\times 1012 / \mathrm{L})$ \\
\hline \multirow{2}{*}{ TEG $(n=266)$} & Preoperation & $126.41 \pm 12.41$ & $8.75 \pm 1.22$ & $238.41 \pm 20.33$ & $3.97 \pm 0.09$ \\
& Postoperation & $106.31 \pm 11.25^{\mathrm{ab}}$ & $10.08 \pm 1.22^{\mathrm{ab}}$ & $149.32 \pm 12.41^{\mathrm{a}}$ & $3.30 \pm 0.06^{\mathrm{ab}}$ \\
\hline \multirow{2}{*}{ CCT $(n=214)$} & Preoperation & $125.32 \pm 12.44$ & $8.26 \pm 1.34$ & $235.26 \pm 20.12$ & $3.98 \pm 0.08$ \\
& Postoperation & $76.56 \pm 8.44^{\mathrm{a}}$ & $14.36 \pm 1.15^{\mathrm{a}}$ & $148.26 \pm 12.41^{\mathrm{a}}$ & $3.11 \pm 0.05^{\mathrm{a}}$ \\
\hline
\end{tabular}

The letter of a suggests $P<0.05$ compared to the CCT group. The letter of $\mathrm{b}$ suggests $P<0.05$ compared to preoperation.

TABLE 3: Changes of coagulation function following transfusion therapy guided by TEG or CCTs.

\begin{tabular}{lcccccc}
\hline Group & Time & PT $(\mathrm{s})$ & APTT $(\mathrm{s})$ & TT $(\mathrm{s})$ & FIB $(\mathrm{g} / \mathrm{L})$ & D-D $(\mathrm{ng} / \mathrm{mL})$ \\
\hline \multirow{2}{*}{ TEG $(n=266)$} & Preoperation & $20.31 \pm 1.06$ & $41.08 \pm 4.64$ & $26.26 \pm 1.31$ & $5.07 \pm 0.05^{\mathrm{ab}}$ & $814.41 \pm 50.33$ \\
& Postoperation & $10.14 \pm 1.22^{\mathrm{ab}}$ & $29.08 \pm 3.22^{\mathrm{ab}}$ & $15.54 \pm 1.26^{\mathrm{ab}}$ & $2.27 \pm 0.09$ & $760.26 \pm 50.33^{\mathrm{a}}$ \\
\hline \multirow{2}{*}{ CCT $(n=214)$} & Preoperation & $20.32 \pm 1.11$ & $41.06 \pm 4.11$ & $26.33 \pm 2.17$ & $5.09 \pm 0.05^{\mathrm{ab}}$ & $814.26 \pm 50.29$ \\
& Postoperation & $13.31 \pm 2.18^{\mathrm{a}}$ & $33.61 \pm 4.33^{\mathrm{a}}$ & $18.64 \pm 1.21^{\mathrm{a}}$ & $3.33 \pm 0.04^{\mathrm{a}}$ & $783.36 \pm 50.41^{\mathrm{a}}$ \\
\hline
\end{tabular}

The letter of a suggests $P<0.05$ compared to the CCT group. The letter of $\mathrm{b}$ suggests $P<0.05$ compared to pre-operation.

the $t$-test. A level of $P<0.05$ suggests a statistically significant difference.

\section{Results}

3.1. Reduced Blood Products by TEG-Guided Transfusion Therapy. The transfusion of RBCs and nonRBC components which involves fresh-frozen plasma, platelet, and cryoprecipitate is critical to hemostasis. The TEG group used fewer volumes of blood products including RBC suspension, fresh-frozen plasma, cryoprecipitate, and apheresis platelets than the CCT group $(P<0.05)(P<0.05$, Table 1$)$. In addition, results showed that the TEG group had a shorter length of hospital stay than the CCT group $(P<0.001$, Table 1$)$.

3.2. Blood Routine Tests following TEG- or CCT-Guided Transfusion Therapy. Prior to orthopedic surgery, there was no significant difference on the value of $\mathrm{Hb}, \mathrm{WBC}, \mathrm{PLT}$, and RBC between the TEG group and CCT group $(P>0.05$ ). As listed in Table 2, after orthopedic surgery, the $\mathrm{Hb}$ and RBC counts were decreased, but the WBC counts were increased in all patients receiving whether transfusion therapy guided by TEG or CCTs. Importantly, the TEG group exhibited fewer WBC counts concomitant with higher $\mathrm{Hb}$ and more RBC counts than the CCT group $(P<0.05)$. There was no significant difference on PLT counts between the two groups before and after surgery $(P>0.05)$.

3.3. Changes of the Coagulation Function following Transfusion Therapy Guided by TEG or CCTs. There was lit- tle difference on the PT, APTT, TT, FIB, and D-D between the two groups before surgery $(P>0.05)$. Significant declines on TT, PATT, PT, and D-D, along with an increase on FIB were observed in two groups after surgery. The TEG group showed reduced TT, PATT, PT, and D-D and elevated FIB compared to the CCT group, as revealed in Table 3 $(P<0.05)$.

3.4. Changes of Coagulation Indexes on the TEG before and after Transfusion. The following TEG variables are associated with the coagulation function. The $R$ value indicates the time to initial fibrin formation; the $K$ value suggests the time until specific clot strength of $20 \mathrm{~mm}$ obtained; the MA reveals the final strength of the clot; the angle value shows rate of clot formation; the CI is obtained by the calculation of the $R, K$, $\alpha$ and MA. As shown in Table 4 , the $K$ time and $R$ time were significantly shortened, while the values of MA, CI, and angle were increased after transfusion $(P<0.05)$.

3.5. TEG-Guided Transfusion Therapy Prevented Liver Dysfunction. There was slight difference on the levels of ALT, AST, ALB, and TBIL between the TEG and CCT groups before orthopedic surgery $(P>0.05)$. After surgery, increased levels of ALT, AST, and TBIL concomitant with a decreased level of ALB were indicated in the TEG and CCT groups. However, the TEG group showed lower levels of ALT, AST, and TBIL and a higher level of ALB compared to the CCT group $(P<0.05$, Table 5$)$. 
TABLE 4: Changes of coagulation indexes on the TEG before and after transfusion.

\begin{tabular}{|c|c|c|c|c|c|}
\hline Time & $K$ time $(\min )$ & $R$ time (min) & MA value $(\mathrm{mm})$ & $\mathrm{CI}$ & Angle $\left({ }^{\circ}\right)$ \\
\hline Before transfusion & $4.54 \pm 0.03$ & $7.27 \pm 0.07$ & $47.64 \pm 5.11$ & $-2.71 \pm 0.05$ & $53.85 \pm 6.22$ \\
\hline After transfusion & $3.67 \pm 0.05^{\mathrm{b}}$ & $5.96 \pm 0.05^{\mathrm{b}}$ & $57.74 \pm 5.22^{\mathrm{b}}$ & $1.46 \pm 0.22^{\mathrm{b}}$ & $62.71 \pm 6.13^{\mathrm{b}}$ \\
\hline
\end{tabular}

$K$ time: time to reach a certain clot strength; $R$ time: coagulation reaction time; angle $(\alpha)$ : clot formation rate; MA value: maximum amplitude; CIn: coagulation index. The letter of $\mathrm{b}$ suggests $P<0.05$ compared to pretransfusion.

TABLE 5: Changes of liver function indexes between the TEG and CCT groups.

\begin{tabular}{lccccc}
\hline Group & Time & ALT $(\mathrm{U} / \mathrm{L})$ & AST $(\mathrm{U} / \mathrm{L})$ & ALB $(\mathrm{g} / \mathrm{mL})$ & TBIL $(\mu \mathrm{mol} / \mathrm{L})$ \\
\hline \multirow{2}{*}{ TEG $(n=266)$} & Preoperation & $32.27 \pm 3.14$ & $38.41 \pm 3.22$ & $33.31 \pm 3.25$ & $18.47 \pm 1.26$ \\
& Postoperation & $41.82 \pm 4.33^{\mathrm{b}}$ & $65.64 \pm 7.14^{\mathrm{b}}$ & $26.68 \pm 2.33^{\mathrm{b}}$ & $20.49 \pm 2.33^{\mathrm{b}}$ \\
\hline \multirow{2}{*}{ CCT $(n=214)$} & Preoperation & $32.25 \pm 3.11$ & $38.21 \pm 2.36$ & $33.32 \pm 3.09$ & $18.45 \pm 1.17$ \\
& Postoperation & $47.21 \pm 4.19^{\mathrm{b}}$ & $72.59 \pm 5.41^{\mathrm{b}}$ & $21.21 \pm 2.35^{\mathrm{b}}$ & $23.64 \pm 2.17^{\mathrm{b}}$ \\
\hline
\end{tabular}

The letter of $\mathrm{b}$ suggests $P<0.05$ compared to preoperation.

TABLE 6: Changes of renal function indexes between the TEG and CCT groups.

\begin{tabular}{lcccc}
\hline Group & Time & BUN $(\mathrm{mmol} / \mathrm{L})$ & Scr $(\mu \mathrm{mol} / \mathrm{L})$ & $\mathrm{UA}(\mu \mathrm{mol} / \mathrm{L})$ \\
\hline \multirow{2}{*}{ TEG $(n=266)$} & Preoperation & $6.13 \pm 0.12$ & $61.91 \pm 6.41$ & $363.25 \pm 30.17$ \\
& Postoperation & $7.21 \pm 0.11^{\mathrm{b}}$ & $81.95 \pm 6.21^{\mathrm{b}}$ & $314.41 \pm 20.33^{\mathrm{b}}$ \\
\hline \multirow{2}{*}{ CCT $(n=214)$} & Preoperation & $6.13 \pm 0.18$ & $61.93 \pm 6.22$ & $362.19 \pm 31.33$ \\
& Postoperation & $7.69 \pm 0.12^{\mathrm{b}}$ & $96.58 \pm 6.17^{\mathrm{b}}$ & $297.26 \pm 20.33^{\mathrm{b}}$ \\
\hline
\end{tabular}

The letter of $\mathrm{b}$ suggests $P<0.05$ compared to preoperation.

3.6. TEG-Guided Transfusion Therapy Prevented Renal Dysfunction. Permanent renal dysfunction is regarded as a serious complication occurring following major surgery, possibly resulting in increased morbidity and mortality. Considering that, the renal function of patients undergoing orthopedic surgery was compared between the TEG and CCT groups. There was no significant difference on the levels of BUN, Scr, and UA between the TEG and CCT groups before surgery $(P>0.05)$. As listed in Table 6 , there was an increase on the levels of BUN and Scr, with a decline on the level of UA in the two groups after surgery, but the levels of BUN and Scr were lower, and the level of UA was higher in the TEG group than those in the CCT group $(P<0.05)$.

\section{Discussion}

The substantial blood loss happens in the trauma, obstetrical, and surgical surgery [13]. In various clinical settings, the massive bleeding is highly related to the mortality and morbidity, especially for severe trauma patients [14]. The massive blood loss reveals the challenge to the coagulation system [3]. Blood transfusion is a relatively safe but with potential adverse reactions, which might be fatal [15]. Therefore, it is necessary to evaluate thehemostasis function and correct coagulation disorder in time, and an appropriate transfusion management should be made [16].

The TEG as a viscoelastic hemostasis assay which evaluates coagulation and fibrinolysis properties by the use of whole blood samples [17], which measures the $K$ value, $R$ value, the angle value, MA value, and CI, and these coagula- tion parameters are associated with clot factor, platelet count, FIB function, and thrombus risk $[18,19]$. According to the data in this study, we found that the above parameters changed after transfusion, the $K$ value and $R$ value were highly decreased, and the value of MA, CI, and angle was obviously increased, which indicated the increase on clot factors and enhancement on FIB function and platelet. The PT, APTT, TT, and FIB were the basic indexes to judge whether the coagulation function is normal or not [20], and the D-D is related to the fibrinolytic activity [21]. Significant declines on TT, PATT, PT, and D-D, along with an increase on FIB were observed in two groups after surgery. The TEG group showed reduced TT, PATT, PT, D-D, and elevated FIB compared to the CCT group. The study suggested that the FIB value was higher in COVID-19 patients than that in the health group, especially for patients with thrombotic disease. The PT, APTT, D-D, and TT were related to the disease severity [22]. Some studies have shown that the TEG group reduced the consumption of blood products in the surgical surgery and severe coagulopathy [23-25], which were similar to our study, and the usage of RBC suspension, fresh-frozen plasma, cryoprecipitate and apheresis platelet in the TEG group was significantly reduced compared to the CCT group, but a study about liver transplantation indicated that the TEG group did not reduce intraoperative blood product consumption [26]. There should be more data to support above outcome since liver transplantation and orthopedic surgery belong to different types. Blood involves plasma, RBC, WBC, and PLT which represent physiological and pathological conditions of the human body [27] According to the 
routine blood tests, this study revealed that there was an significant decline on the $\mathrm{Hb}$ and $\mathrm{RBC}$ and an increase on the WBC after surgery, but the WBC was lower, $\mathrm{Hb}$ and $\mathrm{RBC}$ were higher in the TEG group than those in the CCT group, and there was no obvious difference on PLT between the two groups, which suggested that the TEG group could reduce the incidence of inflammation and anemia caused by blood loss. Under the guidance of TEG, the liver function of the patients was less damaged. Our data indicated a lower growth on the ALT, AST, and TBIL and a lower decline on the ALB, representing the injury of the liver function. These findings were closer to the other COVID-19 experiments, which suggested that the severe patients were with higher growth on the ALT and AST and slight difference on TBIL between the severe patients and the mild patients [28], and 34\% of patients are with lower ALB, and $6 \%$ of patients were with abnormal TBIL [29]. BUN is closely with the renal function, and the elevated BUN suggests impaired renal function [30]. Scr is widely used as a biomarker of the renal function in clinical [31], and the research found that the rise on Scr caused by drug administration might be related to the impairment on the renal function [32]. UA, excreted mostly by the kidney, might be an essential factor which relates to the acute kidney injury, chronic kidney disease, and hypertension [33]. This study indicated that there was an increase on the levels of BUN and Scr, with a decline on the level of UA in the two groups after surgery, but the levels of BUN and Scr were lower, and the level of UA was higher in the TEG group than those in the CCT group, which implied the TEG-guided transfusion therapy prevented renal dysfunction. Besides the above advantages mentioned in this paper, there are some data limitations, such as the lack of adverse reactions and mortality after blood transfusion. Further studies should be carried out to ensure its efficacy.

In summary, this study suggests that the TEG-guided transfusion strategy is superior to CCT-guided during orthopedic surgery, since TEG-guided transfusion therapy could reduce use of blood products, optimize blood components, and improve coagulation function for patients undergoing orthopedic surgery. Importantly, TEG-guided transfusion therapy may prevent liver and renal dysfunction after orthopedic surgery. However, this study was a noninterventional, observational study and did not have the capacity to allow outcome-based comparisons to determine the clinical value of TEG over CCTs or assess TEG as a predictor of bleeding or thrombosis. Therefore, further investigation with larger sample size is required.

\section{Data Availability}

The data used to support the findings of this study are included within the article.

\section{Conflicts of Interest}

The author(s) declare(s) that they have no conflicts of interest.

\section{References}

[1] Z. X. Lin and S. K. Woolf, "Safety, efficacy, and costeffectiveness of tranexamic acid in orthopedic surgery," Orthopedics, vol. 39, no. 2, pp. 119-130, 2016.

[2] J. Xie, X. Feng, J. Ma et al., "Is postoperative cell salvage necessary in total hip or knee replacement? A meta-analysis of randomized controlled trials," International Journal of Surgery, vol. 21, pp. 135-144, 2015.

[3] J. E. Bible, M. Mirza, and M. A. Knaub, "Blood-loss management in spine surgery," The Journal of the American Academy of Orthopaedic Surgeons, vol. 26, no. 2, pp. 35-44, 2018.

[4] E. Guerado, A. Medina, M. I. Mata, J. M. Galvan, and M. L. Bertrand, "Protocols for massive blood transfusion: when and why, and potential complications," European Journal of Trauma and Emergency Surgery, vol. 42, no. 3, pp. 283-295, 2016.

[5] L. M. Carabini, C. Zeeni, N. C. Moreland et al., "Development and validation of a generalizable model for predicting major transfusion during spine fusion surgery," Journal of Neurosurgical Anesthesiology, vol. 26, no. 3, pp. 205-215, 2014.

[6] Z. K. McQuilten, G. Crighton, S. Brunskill et al., "Optimal dose, timing and ratio of blood products in massive transfusion: results from a systematic review," Transfusion Medicine Reviews, vol. 32, no. 1, pp. 6-15, 2018.

[7] F. Kong, Y. Li, and X. Liu, "Effect and clinical value of coagulation test on adverse reactions of blood transfusion in patients with major bleeding in ectopic pregnancy," Experimental and Therapeutic Medicine, vol. 16, no. 6, pp. 4712-4716, 2018.

[8] M. P. Sepulveda, U. A. Salgado, G. J. Barriga et al., "Usefulness of the thromboelastogram in children: correlation with habitual coagulation tests," Revista Chilena de Pediatría, vol. 90, no. 6, pp. 617-623, 2019.

[9] H. Hartert, "Blood clotting studies with thrombus stressography; a new investigation procedure," Klinische Wochenschrift, vol. 26, no. 37-38, pp. 577-583, 1948.

[10] M. Othman and H. Kaur, "Thromboelastography (TEG)," Methods in Molecular Biology, vol. 1646, pp. 533-543, 2017.

[11] L. Shore-Lesserson, H. E. Manspeizer, M. DePerio, S. Francis, F. Vela-Cantos, and M. A. Ergin, "Thromboelastographyguided transfusion algorithm reduces transfusions in complex cardiac surgery," Anesthesia and Analgesia, vol. 88, no. 2, pp. 312-319, 1999.

[12] D. Bolliger and K. A. Tanaka, "Point-of-care coagulation testing in cardiac surgery," Seminars in Thrombosis and Hemostasis, vol. 43, no. 4, pp. 386-396, 2017.

[13] H. P. Pham and B. H. Shaz, "Update on massive transfusion," British Journal of Anaesthesia, vol. 111, Suppl 1, pp. i71-i82, 2013.

[14] A. M. Abuzeid and T. O’Keeffe, "Review of massive transfusion protocols in the injured, bleeding patient," Current Opinion in Critical Care, vol. 25, no. 6, pp. 661-667, 2019.

[15] C. Atterbury and J. Wilkinson, "Blood transfusion," Nursing Standard, vol. 14, no. 34, pp. 47-52, 2000.

[16] M. Munoz, J. Stensballe, A. S. Ducloy-Bouthors et al., "Patient blood management in obstetrics: prevention and treatment of postpartum haemorrhage. A NATA consensus statement," Blood Transfusion, vol. 17, no. 2, pp. 112-136, 2019.

[17] N. Tekkesin, M. Tekkesin, and G. Kaso, “Thromboelastography for the monitoring of the antithrombotic effect of lowmolecular-weight heparin after major orthopedic surgery," 
Anatolian Journal of Cardiology, vol. 15, no. 11, pp. 932-937, 2015.

[18] J. C. Hagedorn 2nd, J. M. Bardes, C. L. Paris, and R. W. Lindsey, "Thromboelastography for the orthopaedic surgeon," The Journal of the American Academy of Orthopaedic Surgeons, vol. 27, no. 14, pp. 503-508, 2019.

[19] E. Gonzalez, E. E. Moore, and H. B. Moore, "Management of trauma-induced coagulopathy with thrombelastography," Critical Care Clinics, vol. 33, no. 1, pp. 119-134, 2017.

[20] G. M. Zhang, W. Zhang, and G. M. Zhang, "Age-specific reference intervals for PT, aPTT, fibrinogen and thrombin time for parturient women," Thrombosis and Haemostasis, vol. 119, no. 6, pp. 894-898, 2019.

[21] J. I. Weitz, J. C. Fredenburgh, and J. W. Eikelboom, "A test in context: D-dimer," Journal of the American College of Cardiology, vol. 70, no. 19, pp. 2411-2420, 2017.

[22] X. Jin, Y. Duan, T. Bao et al., "The values of coagulation function in COVID-19 patients," PLoS One, vol. 15, no. 10, article e0241329, 2020.

[23] J. D. Dias, A. Sauaia, H. E. Achneck, J. Hartmann, and E. E. Moore, "Thromboelastography-guided therapy improves patient blood management and certain clinical outcomes in elective cardiac and liver surgery and emergency resuscitation: a systematic review and analysis," Journal of Thrombosis and Haemostasis, vol. 17, no. 6, pp. 984-994, 2019.

[24] C. F. Weber, K. Gorlinger, D. Meininger et al., "Point-of-care testing: a prospective, randomized clinical trial of efficacy in coagulopathic cardiac surgery patients," Anesthesiology, vol. 117, no. 3, pp. 531-547, 2012.

[25] L. De Pietri, M. Bianchini, R. Montalti et al., "Thrombelastography-guided blood product use before invasive procedures in cirrhosis with severe coagulopathy: a randomized, controlled trial," Hepatology, vol. 63, no. 2, pp. 566-573, 2016.

[26] R. Gaspari, L. Teofili, P. Aceto et al., “Thromboelastography does not reduce transfusion requirements in liver transplantation: a propensity score-matched study," Journal of Clinical Anesthesia, vol. 69, p. 110154, 2021.

[27] D. H. Kuan, C. C. Wu, W. Y. Su, and N. T. Huang, "A microfluidic device for simultaneous extraction of plasma, red blood cells, and on-chip white blood cell trapping," Scientific Reports, vol. 8, no. 1, p. 15345, 2018.

[28] F. Lei, Y. M. Liu, F. Zhou et al., "Longitudinal association between markers of liver injury and mortality in COVID-19 in China," Hepatology, vol. 72, no. 2, pp. 389-398, 2020.

[29] X. Deng, B. Liu, J. Li, J. Zhang, Y. Zhao, and K. Xu, "Blood biochemical characteristics of patients with coronavirus disease 2019 (COVID-19): a systemic review and meta-analysis," Clinical Chemistry and Laboratory Medicine, vol. 58, no. 8, pp. 1172-1181, 2020.

[30] M. Seki, M. Nakayama, T. Sakoh et al., "Blood urea nitrogen is independently associated with renal outcomes in Japanese patients with stage 3-5 chronic kidney disease: a prospective observational study," BMC Nephrology, vol. 20, no. 1, p. 115, 2019.

[31] T. D. Bjornsson, "Use of serum creatinine concentrations to determine renal function," Clinical Pharmacokinetics, vol. 4, no. 3, pp. 200-222, 1979.
[32] X. Chu, K. Bleasby, G. H. Chan, I. Nunes, and R. Evers, "The complexities of interpreting reversible elevated serum creatinine levels in drug development: does a correlation with inhibition of renal transporters exist?," Drug Metabolism and Disposition, vol. 44, no. 9, pp. 1498-1509, 2016.

[33] S. A. Fathallah-Shaykh and M. T. Cramer, "Uric acid and the kidney," Pediatric Nephrology, vol. 29, no. 6, pp. 999-1008, 2014. 